\title{
In-situ formation of carbon shells on the surface of Ni nanoparticles synthesized by the electric explosion of wire
}

\author{
I. V. Beketov ${ }^{1,2}$, A.P. Safronov ${ }^{1,2}$, A. I. Medvedev ${ }^{1,2}$, A. M. Murzakaev ${ }^{1,2}$, \\ O. R. Timoshenkova ${ }^{1}$, T. M. Demina ${ }^{1}$ \\ ${ }^{1}$ Institute of Electrophysics UB of RAS, 620016, Yekaterinburg, Amundsen st., 106, Russia \\ ${ }^{2}$ Ural Federal University, 620002, Yekaterinburg, Mira st., 19, Russia \\ beketov@iep.uran.ru
}

DOI 10.17586/2220-8054-2018-9-4-513-520

The controlled addition of butane to the inert working gas during the production of nickel nanoparticles by the electrical explosion of wire (EEW) method leads to the formation of carbon shells on the surface of particles. EEW provides formation of spherically shaped nanoparticles, with an average diameter that varies from 60 to $100 \mathrm{~nm}$ and depended on the energy introduced into the wire in the EEW process. The thickness and the structure of carbon layer deposited onto the surface of Ni nanoparticles as a function of butane addition was characterized by low-temperature adsorption of nitrogen, x-ray diffraction, complex thermoanalysis, transmission and scanning electron microscopy. It was shown that the thickness of carbon shell on the surface of nanoparticles varied from 2 to $6 \mathrm{~nm}$ and depended on the amount of energy introduced into the wire during the EEW process and on the amount of butane added. The crystalline structure of the carbon shell consisted of amorphous and graphite regions.

Keywords: nickel nanoparticles, electrical explosion of wire, core shell, carbon deposition.

Received: 12 July 2018

Revised: 27 July 2018

\section{Introduction}

Ultrafine metal powders have high chemical activity. As the particle size decreases, their reactivity at low temperatures increases, and it greatly complicates their further use. Usually, after the synthesis of the metal nanoparticles they are inactivated by a thin layer of oxide formed on the surface of the particles, which subsequently protects the metal from rapid oxidation. Given a high specific surface area of the powder, even if the oxide layer is thin, the oxide content in the powder is large. Additionally, some metals such as copper [1], do not form a gas-tight oxide shell during inactivation. In order to protect metal nanoparticles from oxidation, their surface can be covered with silicon carbide, polyethylene, and carbon [2], which are not active at low temperatures.

Deposition of the protective carbon layer is the most universal route for passivation. Metal nanoparticles coated with a protective carbon shell can be used in magnetic fluids, sensor devices, hydrogen sorbents, protective coatings, in some biomedical applications and others.

Different methods are used to create a carbon protective shell on the surface of the particles. In particular they are: encapsulation of particles during annealing of metal powder under a hydrocarbon (gasoline) atmosphere [3], filling of carbon tubes with metal [4], annealing of a mixture of metal particles with carbon [5]. All these technologies involve processing as the subsequent next step after the synthesis of nanoparticles. The formation of a carbon shell on the surface of the metal particles in-situ, in the process of their synthesis is, in our opinion, more preferable. One of these techniques is the method of microarc discharge under an inert atmosphere containing hydrocarbon [6] or in the atmosphere of pure hydrocarbon [7]. However, the disadvantage of the methods described in the literature, is their low productivity, often not exceeding 10 grams per hour.

The electric explosion of wire (EEW) is an efficient method of producing the weakly aggregated nanoparticles of metals, alloys and their chemical compounds $[8,9]$. The basic principle of the method is the evaporation of the metal wire between two working electrodes under the electric discharge. The electrical current of several $\mathrm{kV}$ passes through the wire and instantly overheats it in the explosion-type manner, which gives the name of the method. The cloud of metal vapor originated from the explosion of the wire is then expanding in the surrounding gas giving rise to the condensation of spherical nanoparticles. The process of the electrical explosion of portion by portion continuously fed wire goes up in the step-wise manner The details of the method and a scheme of the EEW apparatus used are given elsewhere [8]. The production rate of this method ranges from 50 to $1000 \mathrm{~g} /$ hour depending on the material of the nanopowder. In addition, the method has low energy consumption, not exceeding $50 \mathrm{~kW} \cdot \mathrm{h} / \mathrm{kg}$, is environmentally friendly, and provides high purity of the produced nanoparticles. The EEW units implemented are compact and do not require special premises. The valuable advantage of EEW 
is its flexibility, which provides the in-situ surface modification of synthesized nanoparticles. In particular, in our previous studies $[10,11]$ we had shown the opportunity of the in-situ carbon deposition on the surface of nickel nanoparticles by the addition of butane in the working gas of a EEW installation. Meanwhile, the effects of the nanoparticles' synthetic conditions on their characteristics have not yet been described.

The objective of the present study was to disclose how to control the deposition of carbon shell on the surface of nickel nanoparticles via varying the synthetic conditions in the process of EEW synthesis. We studied the effect of the excess of the electrical energy introduced in the wire in the EEW process and of the amount of butane added to the working gas on the degree of dispersity of the produced nanoparticles, on the thickness of the carbon shells on their surface, as well as on the morphology of the particles and their structure.

\section{Experimental}

The synthesis of nickel nanoparticles was carried out in the EEW installation described in detail in our previous works $[8,9]$. In the present study, the discharge circuit of the installation had an inductance of $0.4 \mu \mathrm{H}$. The capacitance of the capacitor bank was varied from 3.2 to $4.8 \mu \mathrm{F}$, and the charging voltage - from 20 to $30 \mathrm{kV}$, which provided a variation of the overheating ratio $K$ in the range $1-2.2$ ( $K$ is the ratio of the electric energy introduced into the wire to the sublimation energy of the metal material of the wire). To obtain nanoparticles, a wire of nickel (grade NP2) with a diameter of $0.3 \mathrm{~mm}$, with the length of the explosive wire segment of $88 \mathrm{~mm}$ was used. The wire was fed into the installation step-wise. The explosions were carried out at a frequency of $0.5 \mathrm{~Hz}$.

The gas system of the installation consisted of an explosion chamber, two inertial traps with gas flow rotation by $90^{\circ}$ and $180^{\circ}$, a mechanical (cloth) filter and a fan in serial connection and was filled by circulating argon at a pressure of $0.12 \mathrm{MPa}$. Inertial traps were used to separate large particles, including micron-sized; fine particles were collected in the filter. As it was shown by preliminary experiments, the amount of fine particles in the filter depended on the value of the electric energy introduced into the wire and averaged $85 \%$ of the amount of all the powder produced by the EEW. Only nanoparticles from the filter are considered below as a potential product for further use.

To create carbon shells on the surface of the particles during nanoparticle synthesis, gaseous butane was continuously fed into the apparatus, where it was mixed with the working gas of the installation immediately before entering the explosion chamber. Butane flow rate was varied from $0.3-20 \mathrm{~cm}^{3} / \mathrm{s}$ using an adjustable throttle flow. For the convenience of the characterization of nanoparticles obtained in different regimes, the amount of added butane will further be given in the weight ratio to the weight of the evaporated nickel wire $(Q)$. The value of $Q$ was calculated from the ratio of the butane feed rate and the average feed rate of nickel wire. To find the limits of the efficient passivation of $\mathrm{Ni}$ nano, the collected nanoparticles were optionally passivated after the synthesis by an oxide layer on their surface, while oxygen was supplied to the unit at $0.5 \mathrm{~cm}^{3} / \mathrm{s}$. The passivation process was controlled by a sensor of partial pressure of oxygen. Passivation was terminated when the partial pressure of oxygen in the installation reached $2 \mathrm{kPa}$.

The energy introduced into the wire was determined by the oscillograms of the current pulse passing through the wire segment during EEW. The specific surface area of the produced nanoparticles was determined by lowtemperature nitrogen adsorption (Brunauer-Emmett-Teller (BET) approach) using Micromeritics TriStar 3000 analyzer. X-ray phase analysis (XRD) was performed using Bruker D8 DISCOVER diffractometer in $\mathrm{Cu}_{k \alpha}$ radiation $(\lambda=1.54 \AA)$ with a graphite monochromator on a secondary beam. Diffractogram processing was performed using the built-in software TOPAS 3. High-resolution transmission electron microscopy (HRTEM) was performed using JEOL JEM2100 electron microscope at an accelerating voltage $200 \mathrm{kV}$. The amount of carbon deposited onto nickel nanoparticles was determined by complex thermal analysis using NETZSCH STA409 analyzer with mass-spectrometer. The carbon content in shells was calculated by integrating the peak of carbon dioxide release on a complex thermogram. The mass spectrometer was previously calibrated by introducing a metered amount of gaseous $\mathrm{CO}_{2}$ into the gas line. The error in determining the carbon content in the nanopowder was about $10 \mathrm{wt} . \%$.

\section{Results and discussion}

Figure 1 shows the experimental dependence of the specific surface area of nickel nanoparticles (Ni NPs) collected in the filter setup on the consumption of butane at two values of overheating ratio $K-1.2$ and 2 . It can be noticed that the increase in butane flow rate led to a linear increase in the specific surface area of Ni NPs. The increase in the specific surface area is significant at $K=2$ and less significant at $K=1.2$.

For any value of butane/Ni ratio, the specific surface area of the particles obtained at the overheating ratio $K=2$ are approximately $1.5-2$ times higher than the specific surface area of the particles obtained at the degree 


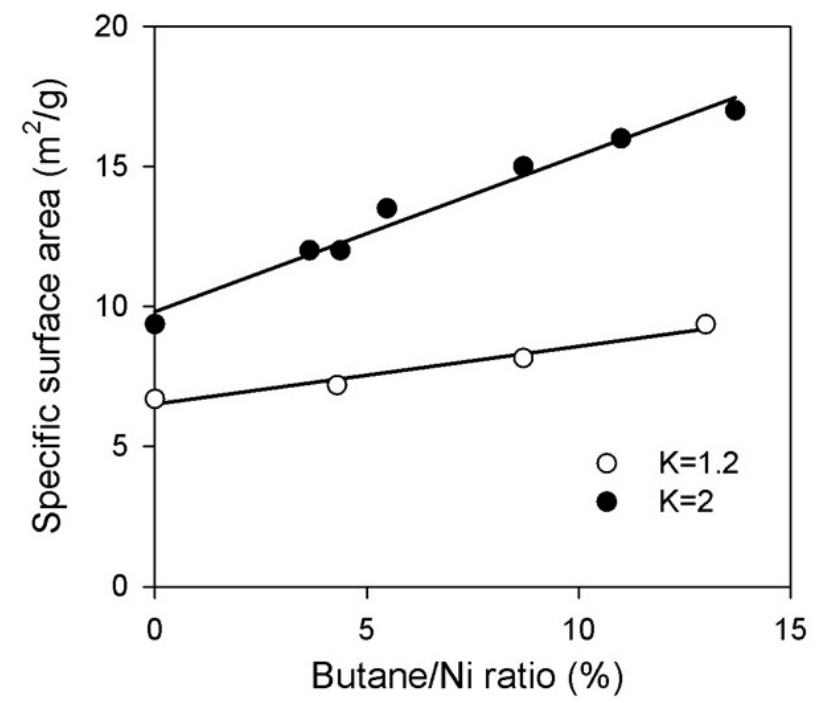

FIG. 1. Dependence of the specific surface area of Ni NPs on butane/Ni ratio $(Q)$ at different overheating ratios $(K)$

of overheating $K=1.2$. This effect is consistent with the literature data [12], which indicate that with an increase in overheating $K$, the size of the obtained particles decreases due to an increase in the expansion rates of the explosion products. As the size of the particles decreases their specific surface area enlarges.

The energy that is released in an electrical explosion is sufficient to decompose the hydrocarbon (butane) into atomic carbon and hydrogen. The temperature of the explosion products at the initial moments of their expansion reaches $10^{4} \mathrm{~K}$ by an order of magnitude. At such temperatures carbon is in a gaseous state. According to the diagram of the state starting from $4000 \mathrm{~K}$ (at normal pressure), carbon condense into graphite [13]. The temperature of graphite formation is higher than the temperature of formation (condensation) of Ni NPs. Thus, the process of formation of the graphite shell can begin from the moment of formation of liquid nickel nanosized droplets. The graphite shell prevents their coagulation and aggregation of metal particles during cooling, and eventually leads to a decrease in their final size and to an increase of the specific surface area of the produced powder.

At high levels of the consumption of hydrocarbon added to the working gas, carbon accumulation as a separate phase is not excluded. The carbon content of the powder samples was determined using a mass spectrometer for $\mathrm{CO}_{2}$ emission during TG analysis. The samples were heated in the air flow to a temperature of $1000{ }^{\circ} \mathrm{C}$ at a heating rate of $10 \mathrm{~K} / \mathrm{min}$. A typical combined thermogram is shown in Fig. 2.

As it can be seen from Fig. 2 (TG curve) the oxidation of nickel (mass gain) starts at $225{ }^{\circ} \mathrm{C}$, ends at $900{ }^{\circ} \mathrm{C}$, and has a clear two-step character with the first step at $225-500{ }^{\circ} \mathrm{C}$ and the second step at $500-900{ }^{\circ} \mathrm{C}$.

The process of $\mathrm{Ni}$ oxidation obscures the reduction of the sample mass due to carbon oxidation to $\mathrm{CO}_{2}$. The use of mass spectrometer allows tracing the process of carbon oxidation contained in the sample. The thermogram shows the peak of the ion current of $\mathrm{CO}_{2}$ emission. Based on the area under the thermogram of ion current, the carbon content in the nanopowder samples obtained at different values of butane/Ni ratios was calculated. Differential scanning calorimetry (DSC) thermogram shows that the processes of oxidation of carbon and nickel are accompanied by exothermic effect. The shape of the thermogram repeats the shape of the mass spectrometric curve of $\mathrm{CO}_{2}$ release with an additional exothermic peak corresponding to the second (high-temperature) stage of nickel oxidation.

Based on the results of thermal analysis, the values of the carbon content in the Ni NPs obtained at different butane/Ni ratios were calculated. The dependence of carbon/Ni ratio in Ni NPs on the butane/Ni ratio in EEW process is reported in Fig. 3. For the sake of clarity, the $\mathrm{x}$-axis, which stands for the butane/Ni ratio in EEW process was recalculated to carbon/ $\mathrm{Ni}$ ratio.

As it can be seen from Fig. 3, the carbon content in the collected Ni NPs detected by the method of complex thermal analysis depends linearly on the amount of carbon introduced (as part of butane) during the EEW of $\mathrm{Ni}$ wire. The carbon content values obtained at different degrees of overheating $K$, lay on a common linear dependence within the experimental error. The tangent of the slope of the line is 1.16 , which is fairly close to unity. This means that all of the carbon introduced into the working gas of the installation during EEW, is almost quantitatively transferred to the Ni NPs synthesized. 


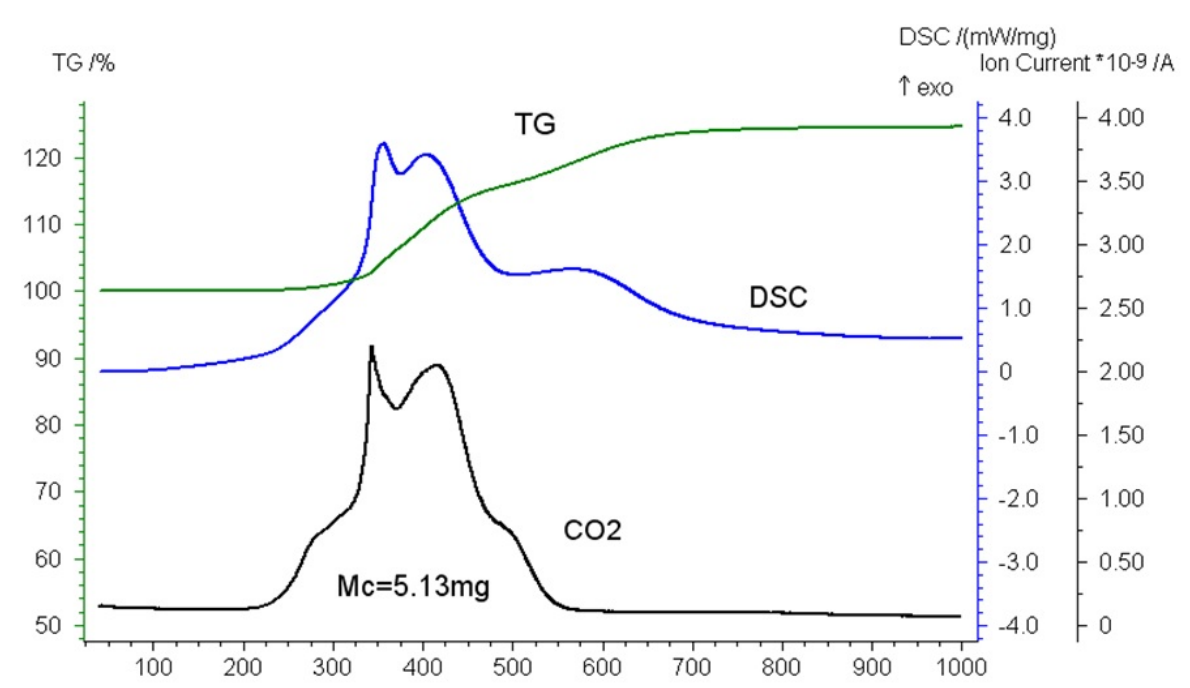

FIG. 2. Combined thermogram of heating in the air of Ni NPs obtained by the EEW at butane/Ni ratio $Q=8.7 \%$. Heating rate $10 \mathrm{~K} / \mathrm{min}$. TG - thermogravimetry curve, DSC - thermogram of differential scanning calorimetry, $\mathrm{CO}_{2}$ - mass-spectrometric signal of carbon dioxide release

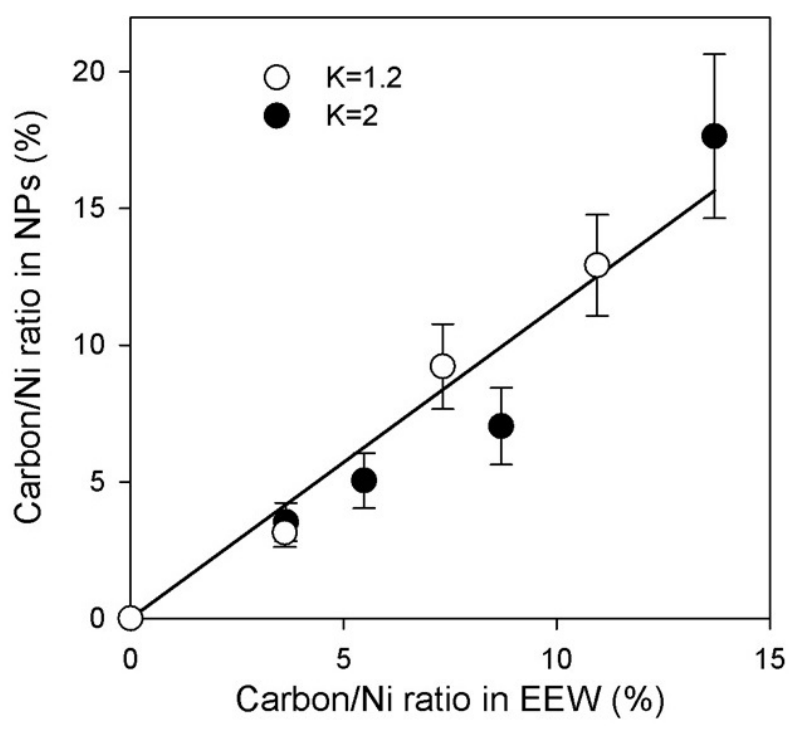

FIG. 3. Comparison of the introduced amount of carbon at EEW at different values of overheating $K$ and the amount of carbon found in the nanopowder by combined thermal analysis

It is known [6] that the metal nanoparticles obtained by the EEW method have a spherical shape, which is a consequence of their condensation in the rarefied vapor phase. The value of the specific surface area for the ensemble of spherical particles, allows calculating their average diameter using the following equation [14]:

$$
d=\frac{6}{S \rho}
$$

where $S$ is the specific surface area, $\rho$ - is the density of the particle material.

Figure 4 reports the calculated values of the average diameter of the particles obtained at different overheating ratios and different butane/ $\mathrm{Ni}$ ratios. In the calculation, according to equation (1), the change in the average density of particles due to an increase in the carbon content in the sample was taken into account.

It is seen that the diameter of the particles obtained at the overheating ratio $K=1.2$ is substantially higher than the diameter of the particles obtained at a higher overheating ratio $K=2$. In general, the average diameter of Ni NPs diminishes with the increase in the amount of carbon introduced into the system at EEW. Meanwhile at $K=1.2$ the change in particle size is insignificant, and at $K=2$ it becomes more pronounced. 


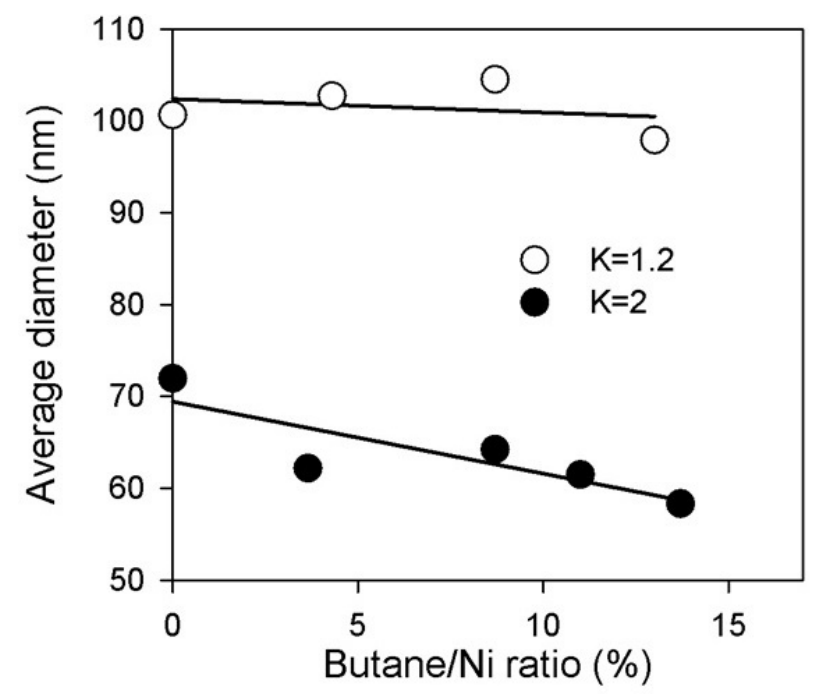

FIG. 4. Dependence of the average particle diameter on the butane/Ni ratio at different degrees of overheating $K$

An XRD pattern of Ni NPs with deposited carbon shell is given in Fig. 5. Diffraction lines are clearly asymmetric and drawn towards small angles, which is possible in the presence of a composition gradient. It may reflect the fact of non-uniform dissolution of carbon in Ni NPs, for example, by the depth of the particle. The crystal lattice period $a=0.3533( \pm 0.0003) \mathrm{nm}$ which differs from the typical value for pure Ni $(a=0.35238 \mathrm{~nm}$, PDF No. 004-08550), also indicates the formation of a solid solution of carbon in Ni NPs. According to reference [15] the maximum solubility of carbon in solid nickel is 2.51 at.\%. Taking into account the atomic weights of carbon and $\mathrm{Ni}$ this value gives $0.52 \mathrm{wt}$. \%) of carbon dissolved in Ni crystal lattice at maximum. This value is substantially lower than the overall carbon content found at Ni NPs at any butane/Ni ratio. Thus we may conclude that the dominating fraction of carbon is deposited on the surface of Ni NPs.

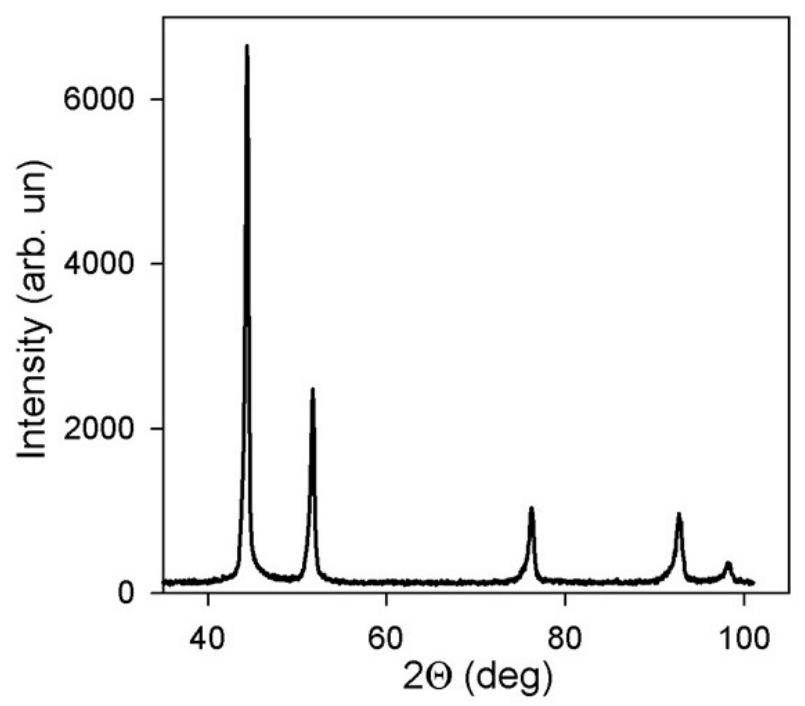

FIG. 5. The diffraction pattern of Ni NPs, obtained at butane/Ni ratio $13 \mathrm{wt} . \%$.

If we assume that all of the particles are covered with a carbon shell of the same thickness, the carbon content in the powder, its specific surface are and the thickness of the shell will be related to each other by the equation:

$$
W_{C}=S \cdot h \cdot \rho_{C},
$$

where $W_{C}$ is the weight fraction of carbon in the Ni nanopowder; $h$ is the thickness of the carbon shell; $\rho_{C}$ is the density of carbon. 
Based on the experimental values of $\mathrm{C}_{c}$ and $\mathrm{S}_{s}$, the thickness of the carbon shell $\mathrm{h}$ on the surface of Ni NPs was calculated using equation (2). Fig. 6 shows the dependence of the calculated thickness of the deposited carbon shell on the value of butane/ $\mathrm{Ni}$ ratio. It is seen that with the increase in the amount of butane supplied to the working gas of the installation, the thickness of the carbon shell on the nickel particles increases. At lower values of overheating ratio $(K=1.2)$ the shells are thicker than carbon shells obtained at overheating ratio $K=2$. Apparently, it is due to the fact that lower $K$ values provide larger particles (see Fig. 4). Given the same overall mass, their number is less than the number of smaller particles obtained at high overheating ratio, so the same amount of carbon is deposited onto smaller surface making the shells on larger particles thicker.

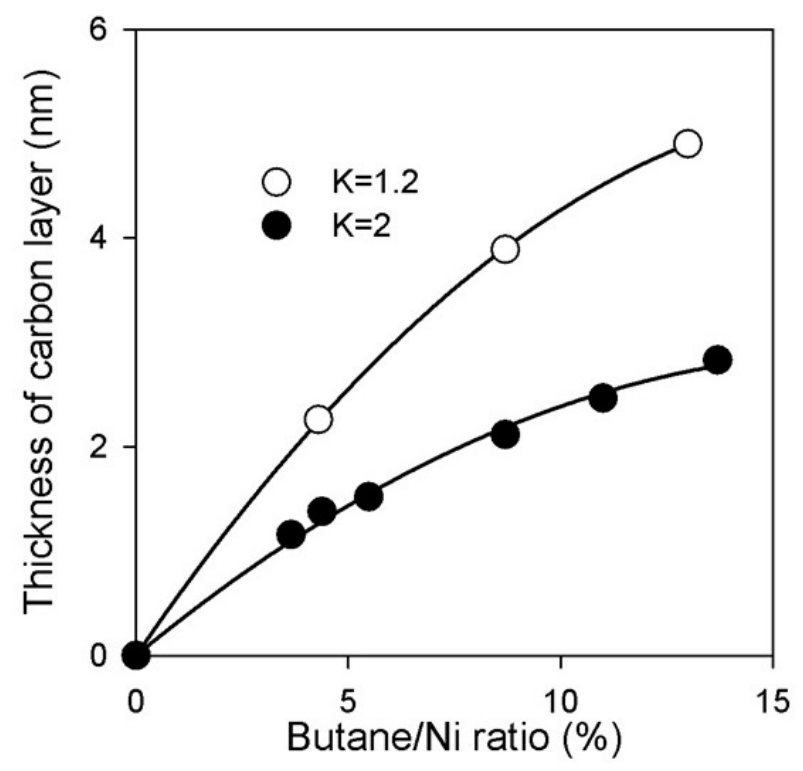

FIG. 6. The dependence of the calculated thickness of the carbon shell on Ni NPs on the butane/Ni ratio at different overheating ratios $(K)$

Figure 7 shows a TEM image of Ni NPs with carbon shells. Deposited carbon shells at the surface of particles are clearly noticeable and their thickness varies from particle to particle. The average thickness of the deposited carbon layer was estimated by the graphical analysis of TEM images. The values are given in Table 1 as a function of butane/Ni ratio and overheating ratio.

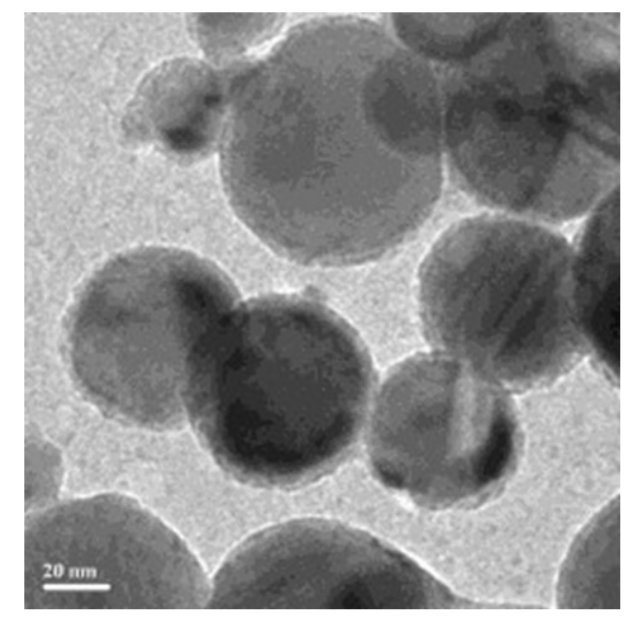

FIG. 7. TEM image of Ni NPs synthesized at butane/Ni ratio of $8.7 \mathrm{wt} . \%$ and overheating ratio $K=1.2$

As it can be seen from Table 1, the increase in butane/Ni ratio leads to an increase in the average thickness of the shell, and the increase in overheating ratio, on the contrary, lowers the average thickness of the shells. These 
TABLE 1. The thickness of the carbon shells based on TEM images

\begin{tabular}{|c|c|c|c|}
\hline Butane/Ni ratio, \% & $K$ & $\begin{array}{c}\text { Average thickness of } \\
\text { the shell, nm }\end{array}$ & Thickness range, nm \\
\hline 4.3 & 1.2 & $3.0 \pm 0.3$ & $2-4$ \\
\hline 13.0 & 1.2 & $4.2 \pm 0.7$ & $2-6$ \\
\hline 3.6 & 2 & $2.7 \pm 0.3$ & $2-4$ \\
\hline 11.0 & 2 & $3.5 \pm 0.5$ & $2-5$ \\
\hline
\end{tabular}

trends and the values of the shell thickness on the surface of nanoparticles are consistent with the data presented above in Fig. 6.

Figure 8 shows the structure of carbon shells obtained by HRTEM. On some particles Fig. 8a presents the evidence of a layered crystalline fragments typical to graphite. The average lattice line spacing was $3.5 \AA$. Meanwhile, the crystal structure is not visible on other particles (Fig. 8b) - possibly, in these cases carbon is in the amorphous state.

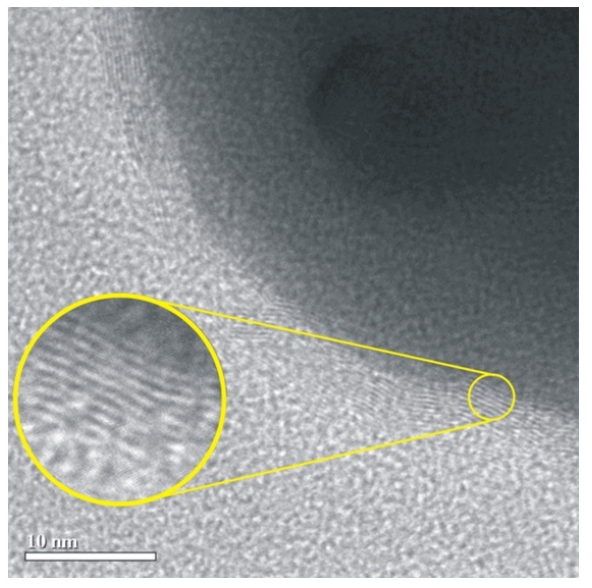

(a)

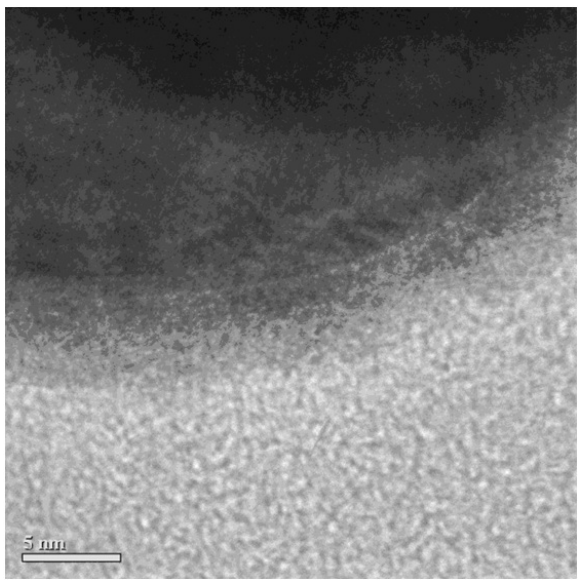

(b)

FIG. 8. The structure of carbon shells on the surface of Ni NPs: a) graphite domains; b) disordered amorphous structures

Judging from the TEM and HRTEM images (Figs. 7 and 8), the deposited carbon forms sufficiently dense shell on the surface of Ni NPs, the thickness of which is estimated as $4-6 \mathrm{~nm}$. Such a shell provides protective coating layer, which prevents oxidation and reduces the pyrophoricity of the $\mathrm{Ni}$ nanopowder as a whole. It was determined experimentally that the minimal butane/Ni ratio, which provides efficient passivation of Ni NPs was $4 \%$. At this butane/Ni ratio the obtained Ni NPs with protective carbon layer can be safely exposed to air.

Meanwhile, if butane/Ni ratio was lower than $4 \%$, the synthesized Ni NPs could be extracted to the air only after passivation with oxygen. Otherwise the samples were flammable. Evidently, the amount of the deposited carbon was not sufficient to cover the total surface of particles in this case. If combined with carbon deposition at low butane/Ni ratio oxygen passivation results in formation of fragments of Ni oxide on the surface of Ni NPs. HRTEM image in Fig. 9 shows such Ni oxide inclusions in the deposited carbon layer. The lattice line spacing of these inclusions was found $4.18 \AA$, which clearly distinguishes them from graphite fragments (Fig. 8a) Eventually, such inclusions operate as "patches" in the non-uniform carbon layer on the surface of Ni NPs.

\section{Conclusion}

Controlled addition of butane $\mathrm{C}_{4} \mathrm{H}_{10}$ to the inert working gas (argon) of the EEW installation during the synthesis of nickel nanoparticles by the electrical explosion of wire provides the deposition of $2-6 \mathrm{~nm}$ dense carbon shells on the surface of spherical nickel nanoparticles from the vapor phase during the condensation process. The energy that is released in an electrical explosion is sufficient to decompose the hydrocarbon (butane) into atomic carbon and hydrogen. Since the temperature of condensation of carbon $(4000 \mathrm{~K})$ is above the temperature 


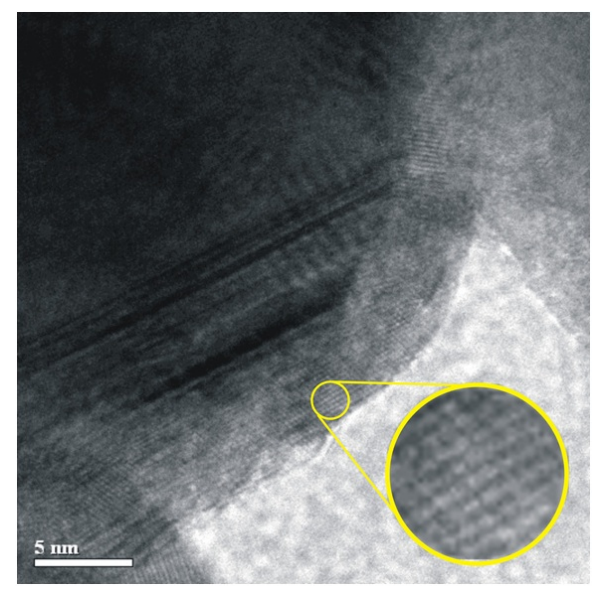

FIG. 9. HRTEM image of the shell on the surface of the Ni NPs synthesized at butane/Ni ratio $2 \%$. Inset - enlarged view of crystal fragment of $\mathrm{Ni}$ oxide

of condensation of $\mathrm{Ni}(3173 \mathrm{~K})$, the process of formation of a graphite shell can begin with the formation of nickel particles. The deposited carbon layer prevents coalescence and aggregation of metal nanoparticles, which leads to a decrease in their size. The increase in butane consumption in the range of butane/Ni ratio $0-10 \%$ results in an increase of the specific surface area of the nanopowder by $40-80 \%$ and in a proportional increase in carbon content. All carbon introduced into the installation is eventually deposited on the nanoparticles. With the increase of the energy introduced into the wire at the EEW, both the diameter of the synthesized nickel nanoparticles and the thickness of the carbon shell on their surface decreases. The structure of the obtained carbon shells includes both fragments of the crystal structure of graphite and disordered carbon in the amorous state. At the same time, the shells are dense enough to prevent oxidation of nickel nanoparticles by aerobic oxygen.

\section{References}

[1] Kotov Yu.A., Rhee Ch.K., Beketov I.V., Bagazeev A.V., Demina T.M., Murzakayev A.M., Samatov O.M., Timoshenkova O.R., Medvedev A.I., Shtols A.K. Production of Copper Nanopowders by Electric Explosion of Wire-Study of Their Oxidation during Storage and Heating in Air. Proc. 9th ISMANAM-2002, J. of Metastable and Nanocrystalline Materials, 2003, 15-16, P. 343-348.

[2] Athanassiou E., Grass R., Stark W. Large-scale production of carbon-coated copper nanoparticles for sensor applications. Nanotechnology, 2006, 17, P. $1668-1673$.

[3] Hayashi C. Ultrafine particles. J. Vac. Sci. Technol. A5, 1987, 4, P. 1375

[4] Tsang S.C., Chen Y.K., Harris P.J.F. Synthesis of carbon nanotubes with totally hollow channels and/or with totally copper filled nanowires. Nature, 1994, 372, P. 159

[5] Tomita S., Hikita M., Fujii M. Formation of Co filled carbon nanocapsules by metal-template graphitization of diamond nanoparticles. $J$. Appl. Phys., 2000, 88, P. 5452.

[6] Ermoline A., Schoenitz M., Dreizin E. and Nan Yao. Production of Carbon-Coated Aluminium Nanopowders in Pulsed Microarc Discharge. Nanotechnology, 2002, 13, P. 638-643.

[7] Zhang Z.D., Zheng J.G., Skorvanek I. Shell/core structure and magnetic properties of carbon-coated Fe-Co(C) nanocapsules. J. Phys. Condens. Matter, 2001, 13, P. 1921

[8] Kotov Yu.A. The electrical explosion of wire: a method for the synthesis of weakly aggregated nanopowders. Nanotechnologies in Russia, 2009, 4(7-8), P. 415-424.

[9] Kotov Yu.A. Electric explosion of wires as a method for preparation of nanopowders. Journal of Nanoparticle Research 5, 2003, P. 539-550.

[10] Beketov I.V., Safronov A.P., Bagazeev A.V., Larranaga A., Kurlyandskaya G.V., Medvedev A.I. In situ modification of Fe and Ni magnetic nanopowders produced by the electrical explosion of wire. Journal of Alloys and Compounds, 2014, 586, P. S483-S488

[11] Safronov A.P., Kurlyandskaya G.V., Chlenova A.A., Kuznetsov M.V., Bazhin D.N., Beketov I.V., Sanchez-Ilarduya M. B., MartinezAmesti A. Carbon deposition from aromatic solvents onto active intact 3d metal surface at ambient conditions. Langmuir, 2014, 30(11), P. 3243-3253

[12] ChaceW.G. A brief survey of exploding wire research. In: Chace W.G. and More H.K. eds. Exploding Wires, Vol. 1. Proc. of 1-st Conf. on the Exploding Wire Phenomenon, Boston, USA, 24-27 March 1959, Plenum Press, New York, P. 7-13.

[13] Properties of elements: Textbook/ under edition of M.E. Drutz. Moscow. Metallurgia. 1985.

[14] Beketov I.V., Safronov A.P., Medvedev A.I., Alonso J., Kurlyandskaya G.V., Bhagat S. M. Iron oxide nanoparticles fabricated by electric explosion of wire: focus on magnetic nanofluids. AIPAdv, 2012, 2, P. 022154.

[15] Gariel A., Chatillon C., and Ansara I. Thermohmial and Phase Diagram Analysis of th Ni-C, Co-C, and Co-Ni-C Systems. High Temperature Science, 1988, 25, P. 17-53. 